\title{
COMMENTARY
}

\section{Metallic nanodevices for chip-scale optical interconnects}

\author{
Liang Tang and David A. B. Miller \\ Ginzton Laboratory, Stanford University, Stanford, CA 94305, USA \\ luketang@gmail.com
}

The use of optics to make connections within and between electronic chips has been the subject of research for over 20 years because it could solve many of the problems experienced in electrical systems [1]. A critical challenge for the convergence of optics and electronics is that the micrometer scale of optics is significantly larger than the nanometer scale of modern electronic devices. In the conversion between photons and electrons, this size incompatibility often leads to substantial penalties in power consumption, area, latency and noise.

Among all the requirements, low power is crucial for chip-scale interconnects. On-chip global interconnects typically consume $1 \mathrm{pJ}$ per bit $(1 \mathrm{~mW} /(\mathrm{Gb} / \mathrm{s}))$. The system energy of optical interconnects needs to be 10 times lower for sufficient advantages over their electrical counterpart. Given the other energies required for the total system, the optical output device (a laser or modulator) should target energies $\sim 10 \%$ of this amount (10 fJ). In such a system we might expect $\sim 10 \%(1 \mathrm{fJ})$ of transmitted energy arrives at the receiving detector, given a reasonable optical loss in the system. To achieve such aggressive energy targets requires miniaturized optoelectronic devices [2].

Metallic nanostructures can confine light to dimensions considerably smaller than the wavelength of the light. They have the potential to play a unique and important role in solving this size-mismatch problem. The strong near fields associated with metallic nanostructures have been studied extensively in recent years. However, until recently, very little research had been done in the interaction of these strong fields with semiconductors, i.e., active metallic nanodevices [3]. Attenuation in metals has limited the propagation length of metallic nanowaveguides to tens of microns, but it has not become a dominating factor for active devices such as light emitters and detectors.

For emitters, the radiative emission rate can be amplified by adopting a small mode volume. Hill et al. reported the first metallic nanolaser emitting at telecom wavelengths with dimensions of 200 to $300 \mathrm{~nm}$ [4]. This electrically pumped nanolaser exhibits a low threshold current smaller than $6 \mu \mathrm{A}$. The structure consists of an $\mathrm{InP} / \mathrm{InGaAs} / \mathrm{InP}$ pillar surrounded by a thin insulating SiN layer encapsulated by gold. This nanolaser has a mode volume of $0.38(\lambda / 2 n)^{3}$, possibly smaller than that of a typical photonic crystal cavity. At low temperatures $(<77 \mathrm{~K})$ where loss in metals is reduced, unambiguous lasing operation was achieved. The $Q$ factor of the metal cavity is very small at room temperature, and therefore lasing was not observed. More recently, a silver cavity with Q factor over 1000 was demonstrated, paving the way for such a laser to operate at room temperature [5].

The receiving end of an optical link typically consists of a detector and an electronic receiver circuit. The detector converts photons into electric current and then the receiver circuit amplifies the current into a large signal suitable for digital logic. If the detector is sufficiently small and thus with a small capacitance, an optical signal can generate large enough voltage swings to drive logic inputs directly from the terminals of the detectors. In this scheme we do not require any receiving amplifier circuit at all, and therefore are 
eliminating the delay, skew, jitter and power dissipation that a normal receiving circuit would introduce.

A photodetector can be made smaller by using a subwavelength active region which, however, could result in very low responsivity because of the diffraction limit of the light. We exploit the idea of a dipole antenna from radio waves, but at near-infrared wavelengths $(\sim 1.3$ $\mu \mathrm{m})$, to concentrate radiation into a nanoscale Ge photodetector in the middle gap of the antenna [6]. The time-varying electric fields associated with light waves exert a force on negatively charged electrons inside the metal and drive them into a collective oscillation. The electron gas is periodically displaced away from the positively charged metallic ions. If the material in the gap is not a conductor, the electrons cannot pass, and charges of opposite signs accumulate on opposing sides of the gap. At specific frequencies, this oscillation is driven resonantly and leads to large charge displacements and high field intensity in the gap. Despite the small antenna size ( $\sim 380 \mathrm{~nm}$ long) and the different properties of metals at such high frequencies $(\sim 230 \mathrm{THz})$, the antenna has qualitatively similar behavior to the common radiofrequency (RF) half-wave Hertz dipole. It gives a relative enhancement of 20 times in the resulting photocurrent in the Ge detector element, which has an active volume of 0.00072 $\mu^{3}$, two orders of magnitude smaller than previously demonstrated detectors at such wavelengths, such as a Si photodiode enhanced by a surface-plasmon antenna [7].

Traditionally metallic nanostructures are investigated with finite-difference time-domain (FDTD) simulation, a time-consuming numerical method for solving the Maxwell equations. FDTD results indicate that the addition of Ge has modified the antenna characteristics considerably. The H-field pattern in Fig. 1(a), corresponding to the scenario with air in the gap, resembles that of two independent dipoles. The H-field in Fig. 1(b), the scenario with Ge in the gap, however, resembles that of a single dipole, indicating that the two dipole arms are strongly coupled together, primarily due to the high refractive index of $\mathrm{Ge}$ in the gap region.
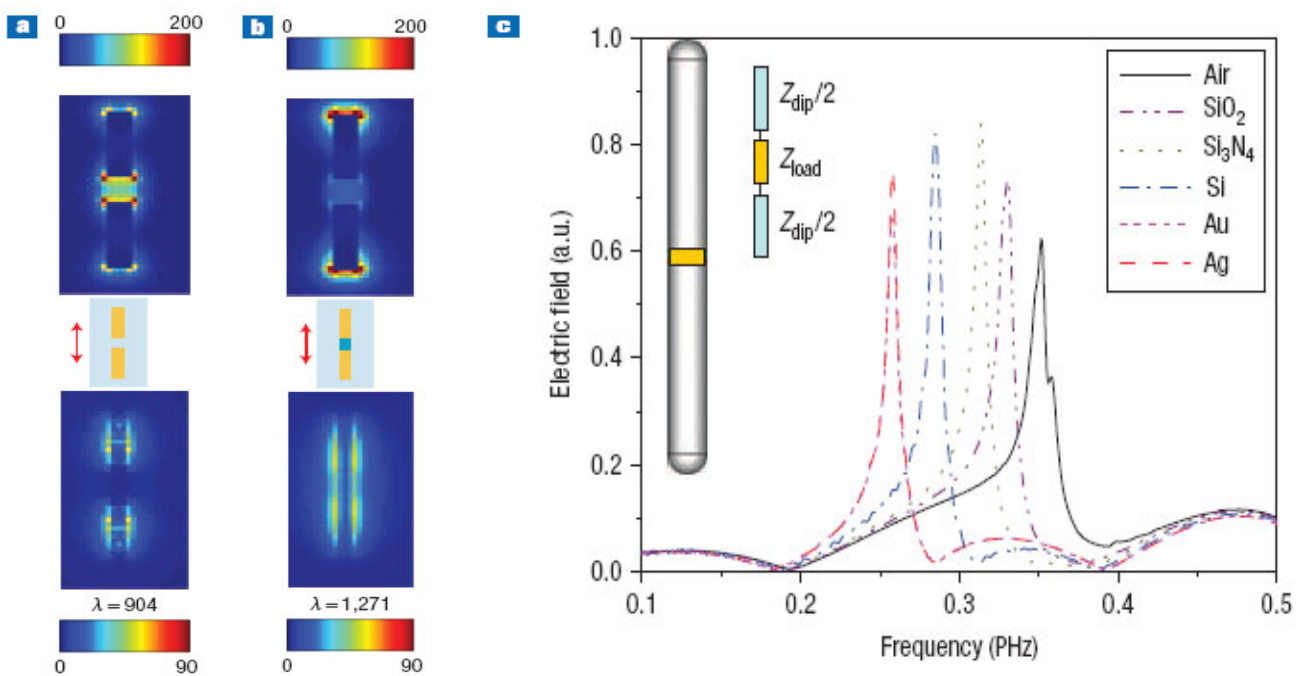

Fig. 1. (a) and (b) are FDTD simulations showing the field pattern and resonant wavelengths without and with Ge [7]. The top row is the E-field intensity, and the bottom row is the H-field intensity. The gold metal arms are all $50 \mathrm{~nm}$ wide, $50 \mathrm{~nm}$ thick and $160 \mathrm{~nm}$ long. The gap between the two metal arms is $60 \mathrm{~nm}$. The substrate is $\mathrm{SiO} 2$. (c) Scattering resonance shift calculated from the circuit model with different materials filling the gap [8]. (C) 2008, Nature Publishing Group. 
Inspired by concepts in the radiofrequency regime, Alù and Engheta have developed a framework to solve antenna problems in the optical regime [8]. By interpreting nanostructures' interaction with light in terms of lumped circuit elements, they have shown that the antenna properties critically rely on the optical properties of the materials inside the gap, and that these can be modeled in a manner analogous to a simple electronic circuit. Insulating nanostructures in the gap can be modeled as capacitors, metals as inductors, and energy dissipation as resistors. A desired frequency response of an optical antenna can now be achieved simply through the optimization of this nanocircuit. This is shown in Fig. 1(c), where the different materials used to fill the gap give rise to a shift in the resonant peak.

In the microwave regime, we often represent waveguiding regions by transmission lines of certain impedances and waveguide discontinuities by lumped circuit elements. By use of a new approach to impedance, transmission line models can be applied to parallel-plate waveguides operating at telecom wavelengths [9]. Each different section of the waveguide is modeled as a transmission line with a characteristic impedance $Z=k /\left(\omega \varepsilon_{\mathrm{d}}\right)$ where $\mathrm{k}$ is the mode propagation number, $\omega$ is the angular frequency and $\varepsilon_{d}$ is the relative permittivity of the dielectric in between the parallel conductors. The impedance-model calculations agree reasonably well with FDTD results and can be used to predict the transmission and reflection of modes several orders of magnitude faster than the FDTD method.

The nanoscale dimensions of the devices raise an experimental challenge, that is, fabricating both the metallic and semiconductor nanostructures with sufficient precision and aligning them with each other. Fortunately, such precision is within the current photolithography capability in the semiconductor industry. Si-based integrated-circuit technology already uses metallic structures, such as $\mathrm{Cu}$ and $\mathrm{Al}$ interconnects, to route electronic signals between transistors on a chip. This mature processing technology can thus be used to our advantage in integrating metallic nanodevices with electronic circuits. Using $\mathrm{Al}$ metal, for the first time, we have demonstrated nanoscale photodetectors fabricated on a commercial Complementary-Metal-Oxide-Semiconductor (CMOS) chip [10].

In summary, significant experimental and theoretical progress has been made in metallic nanodevices. Although the properties of metals are different in the optical regime, antenna and circuit models may provide guidance for future designs. A better physical understanding of metallic nanostructures provided by these models could accelerate the pace of technological advances in chip-scale optical interconnects. In particular, the integration of metallic nanodevices in a CMOS chip presents unique opportunities to solve challenging optoelectronic problems. Some specific on-chip electronics, such as analog-to-digital conversion sampling gates, may be replaced by photonic devices because of their advantages in delay, speed and power consumption.

\section{Acknowledgments}

We appreciate the support of AFOSR "Plasmon Enabled Nanophotonic Circuits" MURI Program and MARCO/DARPA FCRP Interconnect Focus Center.

\section{References}

[1] D. A. B. Miller, "Rationale and challenges for optical interconnects to electronic chips," Proc. IEEE 88, 728-749 (2000) [doi:10.1109/5.867687].

[2] S. Latif, S. E. Kocabas, L. Tang, C. Debaes, and D. A. B. Miller, "Low capacitance CMOS silicon photodetectors for optical clock injection," Appl. Phys. A 95, 813-819 (2009) [doi: 10.1007/s00339-009-5122-5].

[3] L. Tang, D. A. B. Miller, A. K. Okyay, J. A. Matteo, Y. Yuen, K. C. Saraswat, and L. Hesselink, "C-shaped nanoaperture-enhanced germanium photodetector," Opt. Lett. 31, 1519-1521 (2006) [doi:10.1364/OL.31.001519]. 
[4] M. T. Hill, Y. Oei, B. Smalbrugge, Y. Zhu, T. de Vries, P. J. van Veldhoven, F. W. M. van Otten, T. J. Eijkemans, J. P. Turkiewicz, H. de Waardt, E. J. Geluk, S. Kwon, Y. Lee, R. Notzel, and M. K. Smit, "Lasing in metallic-coated nanocavities," Nature Photon. 1, 589-594 (2007) [doi:10.1038/nphoton.2007.182].

[5] B. Min, E. Ostby, V. Sorger, E. Ulin-Avila, L. Yang, X. Zhang, and K. Vahala, "High-Q surface plasmon-polariton whispering-gallery microcavity," Nature 457, 455-458 (2009) [doi:10.1038/nature07627].

[6] L. Tang, S. E. Kocabas, S. Latif, A. K. Okyay, D. Ly-Gagnon, K. C. Saraswat, and D. A. B. Miller, "Nanometre-scale germanium photodetector enhanced by a nearinfrared dipole antenna," Nature Photon. 2, 226-229 (2008) [doi:10.1038/nphoton.2008.30].

[7] T. Ishi, J. Fujikata, K. Makita, T. Baba, and K. Ohashi, "Si nano-photodiode with a surface plasmon antenna," Jap. J. Appl. Phys. 44, L364-L366 (2005) [doi:10.1143/JJAP.44.L364].

[8] A. Alù and N. Engheta, "Tuning the scattering response of optical nanoantennas with nanocircuit loads," Nature Photon. 2, 307-310 (2008) [doi:10.1038/nphoton.2008.53].

[9] S. E. Kocabas, G. Veronis, D. A. B. Miller, and S. Fan, "Transmission line and equivalent circuit models for plasmonic waveguide components," IEEE J. Sel. Top. Quantum Electron. 14, 1462-1472 (2008) [doi:10.1109/JSTQE.2008.924431].

[10] L. Tang, S. Latif, and D. A. B. Miller, "Plasmonic device in Si CMOS," Proc. $21^{\text {st }}$ IEEE Laser. Electro-opt. Soc., 139-140 (2008) [doi:10.1109/LEOS.2008.4688527]. 